\title{
Thermogravimetry and the Negative Temperature Dependence of Gravity
}

\section{Dmitriev AL*}

Department of Mechanics and Optics, St. Petersburg National Research University of Information Technologies, Russia

\section{Introduction}

It is shown that data of thermo gravimetric measurements confirm the negative temperature dependence of gravity. The accounting of this dependence is necessary for increase of accuracy of the thermo gravimetric analysis.

The thermogravimetric analysis based on exact weighing of the heated sample is widely applied in researches of physical and chemical properties of materials [1-3]. The first stage of thermo gravimetric measurements is receiving a basic curve - temperature dependence of weight of the empty holder of a sample, for example, of a crucible. At data processing of temperature measurements the basic curve is subtracted from experimental temperature dependence of weight. Out of areas of change of phase structure of substance in which there are sharp changes of its weight, the main reason for the monotonous increasing temperature dependence of weight is considered action of forces of buoyancy. Meanwhile, physical temperature dependence of weight of bodies [4-6] has essential impact on measurements of weight and has to be taken into account in the exact thermogravimetric analysis; this circumstance was noted by Grumazescu [7]. At creation of a basic curve the seeming mass $M$ of the holder (crucible) in air is equal $M=m-\rho V$, where $m$-the mass of a crucible, $V$-its volume and $\rho$-air density. Temperature change of the seeming weight

$$
\frac{d M}{d t}=\frac{d M}{d t}-\rho \frac{d V}{d t}-V \frac{d \rho}{d t},
$$

Where $V=V_{0}(1+\beta t), \beta$-volume expansion coefficient of material of a crucible. Temperature dependence of $\rho(t)$ is represented by known expression

$$
\rho(t)=\frac{A}{1+B t} \cdot \frac{p}{760},
$$

Where $A=0.0012932 \mathrm{~g} / \mathrm{cm}^{3}, \mathrm{~B}=0.00367 \mathrm{~K}^{-1}, \mathrm{p}$ - air pressure in $\mathrm{mm}$ $\mathrm{Hg}[8]$. By normal ( $p=760)$ air pressure Eq. 1 may be represent as

$$
\frac{d M}{d t}=\frac{d m}{d t}+C_{1}+C_{2}
$$

Where $C_{1=}-V_{0} \beta A(1+\beta \mathrm{t})^{-1}$, and $C_{2=} V_{0}(1+\beta \mathrm{t}) A B$

For numerical estimates we will use results of work [2] according to which the seeming change of mass of a porcelain crucible with the weight $m=4 \mathrm{~g}$ and volume $1.5 \mathrm{~cm}^{3}$ measured on Shevenar's thermo scales in the temperatures range $200-1000^{\circ} \mathrm{C}$ is equal $4.0 .10^{-6} \mathrm{gK}^{-1}$. Volume coefficient of expansion of porcelain $\beta=9.10^{-6} \mathrm{~K}^{-1}[8]$ and, for example, at $\mathrm{t}=200^{\circ} \mathrm{C}$, the values of coefficients $C_{1.2}$ are equal $\mathrm{C}_{1}=-1.0 .10^{-8}$ $\mathrm{gK}^{-1}$ and $C_{2}=7 \cdot 1 \cdot 10^{-6} \mathrm{gK}^{-1}$.

As the absolute values $C_{1} \prec \prec C_{2}$, the main contribution to the seeming change of mass of a crucible is made by the effects of buoyancy described by coefficient $C_{2}$. Obviously, the consent of the experimental and provided settlement data is possible only at $\frac{d m}{d t}=$ $-3.1 .10^{-6} \mathrm{gK}^{-1}$. This fact directly confirms established in [4-6] negative temperature dependence of weight of bodies. Temperature change of weight of the holder, it is generally connected with change of temperature of a porcelain crucible, the assessment of size of relative temperature change of the weight of porcelain from where follows the $\gamma=\frac{d m}{m d t} \approx-0.8 \cdot 10^{-6} \mathrm{~K}^{-1}$. Sign and an order of the specified size correspond to data of measurements of physical temperature dependence of weight of various metals [4-6], and also PZT [9]. For more exact quantitative estimates of influence of temperature dependence of weight on results of thermogravimetric measurements, the accounting of the sizes, forms, masses, physical and thermodynamic characteristics as holder, and the studied sample is necessary $[8,9]$. Temperature dependence of physical weight of bodies will allow to establish the reasons of anomalies of thermogravimetric dependences with bigger degree of reliability and to increase the accuracy of the gravimetric analysis.

\section{References}

1. Wendlandt W (1974) Thermal Methods of Analysis Johns Wiley and Sons

2. Simons EL, Newkirk AE, Aliferis I (1957) Analytical Chemistry 29: 48-54

3. Sestak J (1984) Thermo physical Properties of Solids. Academia Prague.

4. Dmitriev AL, Nikushchenko EM, Snegov VS (2003) Influence of the Temperature of a Body on its Weight, Measurement Techniques. 46: 115-120.

5. Dmitriev AL (2007) Experimental Study of Gravity Force Temperature Dependence. Research Gate

6. Dmitriev AL Bulgakova SA (2013) Negative Temperature Dependence of Gravity is a Reality. Proc. waset 79: 1560-1565.

7. Grumazescu M (2015) On Thermo gravity. Research Gate.

8. Ebert H (1957) Physicalisches Taschenbuch. Springer.

9. Dmitriev AL (2012) Physical substantiation of an opportunity of artificial change of body weight, Physics Procedia 38: 150-163.

${ }^{*}$ Corresponding author: Dmitriev AL, Department of Mechanics and Optics, St. Petersburg National Research University of Information Technologies, Russia, Tel: 812-3154071; E-mail: alex@dmitriyev.ru

Received October 12, 2015; Accepted October 28, 2015; Published October 31 , 2015

Citation: Dmitriev AL (2015) Thermogravimetry and the Negative Temperature Dependence of Gravity. J Phys Math 6: 148. doi:10.4172/2090-0902.1000148

Copyright: @ 2015 Dmitriev AL. This is an open-access article distributed under the terms of the Creative Commons Attribution License, which permits unrestricted use, distribution, and reproduction in any medium, provided the original author and source are credited. 